\title{
THE COSMOS SURVEY: HUBBLE SPACE TELESCOPE ADVANCED CAMERA FOR SURVEYS OBSERVATIONS AND DATA PROCESSING ${ }^{1}$
}

\author{
A. M. Koekemoer,${ }^{2}$ H. Aussel,${ }^{3,4}$ D. Calzetti, ${ }^{2}$ P. Capak,${ }^{5}$ M. Giavalisco, ${ }^{2}$ J.-P. Kneib, ${ }^{6}$ \\ A. Leauthaud, ${ }^{6}$ O. Le Fèvre, ${ }^{6}$ H. J. McCracken, ${ }^{7,8}$ R. Massey, ${ }^{5}$ B. Mobasher ${ }^{2}$ \\ J. Rhodes, ${ }^{5,9}$ N. Scoville, ${ }^{5,10}$ and P. L. Shopbell ${ }^{5}$ \\ Received 2006 November 30; accepted 2007 March 5
}

\begin{abstract}
We describe the details of the Hubble Space Telescope (HST) Advanced Camera for Surveys (ACS) Wide Field Channel (WFC) observations of the COSMOS field, including the data calibration and processing procedures. We obtained a total of 583 orbits of HST ACS/ WFC imaging in the F814W filter, covering a field that is $1.64 \mathrm{deg}^{2}$ in $\operatorname{area}$, the largest contiguous field ever imaged with HST. The median exposure depth across the field is $2028 \mathrm{~s}$ (one HST orbit), achieving a limiting point-source depth $\mathrm{AB}(\mathrm{F} 814 \mathrm{~W})=27.2(5 \sigma)$. We also present details of the astrometric image registration and distortion removal and image combination using MultiDrizzle, motivating the choice of our final pixel scale (30 mas pixel ${ }^{-1}$ ), based on the requirements for weak-lensing science. The final set of images are publicly available through the archive sites at IPAC and STScI, along with further documentation on how they were produced.
\end{abstract}

Subject headings: cosmology: observations — dark matter — galaxies: evolution — galaxies: formation large-scale structure of universe - surveys

\section{INTRODUCTION}

The Cosmic Evolution Survey (COSMOS; Scoville et al. 2007a) is the largest contiguous survey ever undertaken with the Hubble Space Telescope (HST), imaging a $\sim 2 \mathrm{deg}^{2}$ equatorial field with the primary goal of addressing the coupled evolution of largescale structure, star formation, and galaxy activity.

A key requirement for this type of cosmological survey is sensitive imaging at the highest possible angular resolution, particularly for all sources above $z \sim 0.5-1$, where an angular resolution $\lesssim 0.1^{\prime \prime}$ becomes crucial to resolving structure on subkiloparsec scales.

\footnotetext{
1 Based on observations with the NASA/ESA Hubble Space Telescope, obtained at the Space Telescope Science Institute, which is operated by the Association of Universities for Research in Astronomy (AURA), Inc., under NASA contract NAS 5-26555; also based on data collected at: the Subaru Telescope, which is operated by the National Astronomical Observatory of Japan; the XMM-Newton, an ESA science mission with instruments and contributions directly funded by ESA Member States and NASA; the European Southern Observatory under Large Program 175.A-0839, Chile; Kitt Peak National Observatory, Cerro Tololo Inter-American Observatory, and the National Optical Astronomy Observatory, which are operated by AURA under cooperative agreement with the National Science Foundation; the National Radio Astronomy Observatory which is a facility of the National Science Foundation operated under cooperative agreement by Associated Universities, Inc; and the Canada-France-Hawaii Telescope (CFHT) with MegaPrime/MegaCam operated as a joint project by the CFHT Corporation, CEA/DAPNIA, the National Research Council of Canada, the Canadian Astronomy Data Centre, the Centre National de la Recherche Scientifique de France, TERAPIX, and the University of Hawaii.

2 Space Telescope Science Institute, 3700 San Martin Drive, Baltimore, MD 21218.

3 Institute for Astronomy, 2680 Woodlawn Drive, University of Hawaii, Honolulu, HI 96822.

4 Service d'Astrophysique, CEA/Saclay, F-91191 Gif-sur-Yvette, France.

5 California Institute of Technology, MC 105-24, 1200 East California Boulevard, Pasadena, CA 91125.

${ }^{6}$ Laboratoire d'Astrophysique de Marseille, BP 8, Traverse du Siphon, F-13376 Marseille Cedex 12, France.

7 Institut d'Astrophysique de Paris, UMR7095 CNRS, Universitè Pierre et Marie Curie, 98 bis Boulevard Arago, 75014 Paris, France.

8 Observatoire de Paris, LERMA, 61 Avenue de l'Observatoire, F-75014 Paris, France.

9 Jet Propulsion Laboratory, Pasadena, CA 91109.

10 Visiting Astronomer, University of Hawaii, 2680 Woodlawn Drive, Honolulu, HI 96822.
}

This approach was pioneered in the Hubble Deep Fields-North and South (HDF; Williams et al. 1996, 2000), which achieved this level of angular resolution, albeit over a relatively small $5 \operatorname{arcmin}^{2}$ field. Subsequent surveys included the HST Ultra Deep Field (UDF; Beckwith et al. 2006), which went deeper over a somewhat larger $11 \operatorname{arcmin}^{2}$ area; the Great Observatories Origins Deep Survey (GOODS; Giavalisco et al. 2004), which was shallower but covered a larger $360 \mathrm{arcmin}^{2}$ area; and two larger and shallower surveys, each covering $\sim 700 \operatorname{arcmin}^{2}$ (GEMS, Rix et al. 2004; AEGIS, Davis et al. 2006). COSMOS provides an additional factor $\sim 10$ increased coverage in area, and uses broader filters to reach deeper sensitivities, which are crucial for obtaining the accurate galaxy-morphology measurements required to construct mass maps based on weak lensing.

In addition to the $H S T$ data, the COSMOS survey also contains a wealth of ancillary data obtained at other ground-based and space-based telescopes, including X-ray, infrared, submillimeter, and radio imaging, as well as comprehensive spectroscopic programs, which are all summarized in Scoville et al. (2007a). The present paper describes the details of the HST observations and data processing that we used to construct the imaging data sets that form the foundation of the majority of the work in the COSMOS survey; see Scoville et al. (2007b) for an overview.

\section{ACS OBSERVATIONS}

\subsection{ACS Tiling Strategy for the Full COSMOS Mosaic}

The COSMOS survey was awarded a total of 590 orbits of HST observations to cover a large, contiguous field of "tiles" centered at R.A. $=10^{\mathrm{h}} 00^{\mathrm{m}} 28.6^{\mathrm{s}}$, decl. $=+02^{\circ} 12^{\prime} 21.0^{\prime \prime}(\mathrm{J} 2000.0)$ using the Advanced Camera for Surveys (ACS) Wide-Field Channel (WFC) detector, together with the F814W ("Broad I") filter. The ACS/WFC is a mosaic detector consisting of two $2048 \times 4096$ pixel CCDs with $15 \mu \mathrm{m}$ pixels, corresponding to a spatial scale of $\sim 0.05^{\prime \prime} \mathrm{pixel}^{-1}$ and a field of view of $\sim 202^{\prime \prime} \times 202^{\prime \prime}$. The detectors have a read noise of $5 e^{-}$pixel $^{-1}$ and a dark current rate of $0.0038 e^{-} \mathrm{s}^{-1}$ pixel $^{-1}$; the observations were all obtained with a gain of 1, corresponding to a full-well depth of 84,700 electrons (thus, above the maximum A-to-D conversion limit of 


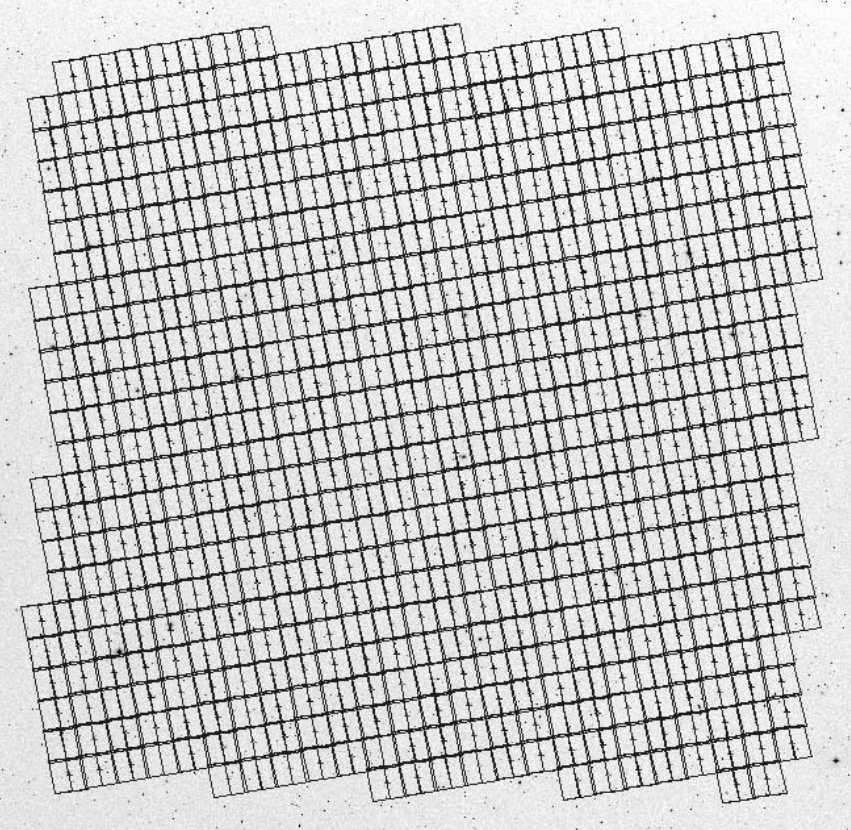

FIG. 1.-Final layout of the full mosaic of all the ACS/WFC F814W pointings, covering a total area of $1.64 \mathrm{deg}^{2}$, or about $77^{\prime}$ on a side. The field is centered at R.A. $=10^{\mathrm{h}} 00^{\mathrm{m}} 28.6^{\mathrm{s}}$, decl. $=+02^{\circ} 12^{\prime} 21.0^{\prime \prime}(\mathrm{J} 2000.0)$; the rectangle fully enclosing all the ACS imaging has lower left and upper right corners (R.A., decl. $[\mathrm{J} 2000.0])$ at $\left(150.7988^{\circ}, 1.5676^{\circ}\right)$ and $\left(149.4305^{\circ}, 2.8937^{\circ}\right)$.

65,535 counts per pixel). The camera is mounted $\sim 6^{\prime}$ off-axis from the principal optical axis of HST and is also tilted with respect to the focal plane of $H S T$, resulting in a significant amount of skew distortion across the field, $\sim 7 \%-10 \%$ of the detector size. Hence, the pixels are projected as skew trapezoids on the sky, with their shapes changing across the detector, and this distortion is removed during postobservation processing, described below.

Due to HST scheduling constraints, the observing program had to be divided between two HST observing cycles from 2003 May to 2005 June, with 270 orbits allocated during Cycle 12 (HST Program ID 9822) and 320 orbits allocated during Cycle 13 (HST Program ID 10092). In order to maximize the amount of contiguous area coverage, it was required that all the tiles be obtained at the same orientation, thereby minimizing the amount of overlap between tiles to less than a few percent. Combining this with additional constraints on the orientation of $H S T$ with respect to the Sun led to all the tiles being tilted about $10^{\circ}$ from north (see Table 1). This need to keep the edges of the full field oriented approximately north-south and east-west (to maximize overlap with ancillary observations from other observatories) led to slightly staggered edges, as shown in Figure 1. There are typically up to 16 orbits available per day on HST; since the allowable roll angles of HST change throughout the year, not all the tiles could be obtained at the same orientation; some of them had to be obtained at orientations that were rotated $180^{\circ}$ relative to the default orientation. In addition, approval was granted to use 9 of the 590 orbits to obtain F475W observations (SDSS $g$ band) of a $3 \times 3$ grid at the field center, as a pilot program to provide a demonstration of the value that two-filter HSTACS/WFC imaging would have for the main scientific goals of the project. Finally, two of the tile pointings failed due to problems with guide stars and were repeated, while two more tiles had severe problems with scattered light from adjacent stars and had to be repeated using slightly different pointings, offset from the original pointing by half a field in both directions (for which we used other orbits from the original total alloca-
TABLE 1

COSMOS HST ACS/WFC OBSERVATIONS

\begin{tabular}{|c|c|c|}
\hline Range of Dates & $\begin{array}{l}\text { P.A. } \\
\text { (deg) }\end{array}$ & $\begin{array}{c}\text { Number of } \\
\text { Pointings }\end{array}$ \\
\hline 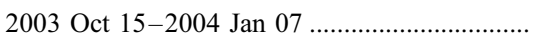 & 100 & 42 \\
\hline 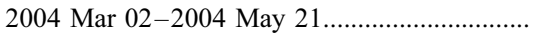 & 280 & 303 \\
\hline 2004 Oct 13-2005 Jan 07 ................................... & 100 & 103 \\
\hline 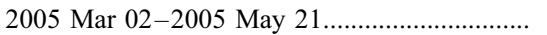 & 280 & 142 \\
\hline 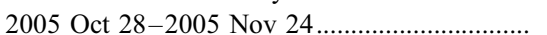 & 100 & $2^{\mathrm{a}}$ \\
\hline
\end{tabular}

a These two additional orbits were awarded to compensate for two pointings lost due to guide-star failures.

tion). Thus, the final contiguous area is $1.64 \mathrm{deg}^{2}$, or approximately $77^{\prime}$ along each side, and contains data from a total of 583 orbits of HST ACS/WFC F814W imaging (including two additional orbits for repeat observations), with an additional nine orbits of F475W imaging at the field center.

\subsection{Cosmic-Ray Splitting and Dither Strategy for Each Tile}

The total ACS/WFC exposure time obtained in F814W for each tile was $2028 \mathrm{~s}$. Since cosmic rays impact between $\sim 2 \%$ and $6 \%$ of the pixels on the ACS detectors during this length of exposure time, the observations for each tile were split into four equal-length exposures, each $507 \mathrm{~s}$ in duration, ensuring that less than 1 pixel out of $4096^{2}$ would be impacted by cosmic rays in all four exposures, according to statistical binomial probability. The exposure time of $507 \mathrm{~s}$ was the maximum that could be achieved within the nominal orbital duration of HST, after accounting for overheads due to readout, telescope motion, and guide-star acquisition.

The four $507 \mathrm{~s}$ exposures for each tile were obtained using a "dither" pattern designed to improve the sampling of the point spread function (PSF), as well as covering the $\sim 3^{\prime \prime}$ gap that is present between the two CCDs of the ACS/WFC. In the F814W filter, the intrinsic width of the PSF that is produced by the $H S T$ optics is $\sim 0.085^{\prime \prime}$. However, this is significantly undersampled by the $0.05^{\prime \prime}$ pixels of the ACS/WFC detector, and the final measured PSF in the combined images tends to be closer to $\sim 0.1^{\prime \prime}$ as a result of convolution by the detector pixels, as well as the pixel size of the final image. The undersampling can be mitigated to some extent by offsetting the detector in a dither pattern that provides subsampling of the PSF in different parts of each pixel during each exposure. As a consequence of the changing pixel size produced by the strong distortion of the ACS/WFC detectors, a given dither offset in arcseconds actually corresponds to different offsets in terms of pixels, and these offsets gradually vary across the detector, further modulating the subsampling pattern achieved with four exposures.

We chose a dither pattern designed to produce optimal subsampling of the PSF by ensuring that any given point would always be sampled by a different part of each detector pixel in the four exposures. This is achieved by offsetting the telescope in integer-pixel and half-pixel increments along both the $x$ and $y$ axes of the detector; thus, the combination of four such offsets ensures that any given point on the sky is sampled by all four quadrants of a pixel during the four exposures. In addition, we added a $\sim 3^{\prime \prime}$ offset along the $y$-direction of the dither pattern in order to cover the gap between the chips, as well as an offset of a few pixels along the $x$-direction of the dither pattern in order to ensure that bad columns and other defects were moved to a different part of the sky during each of the four exposures. The resulting dither pattern is shown in Figure 2, demonstrating how we implemented this using a combination of a primary two-point dither pattern 


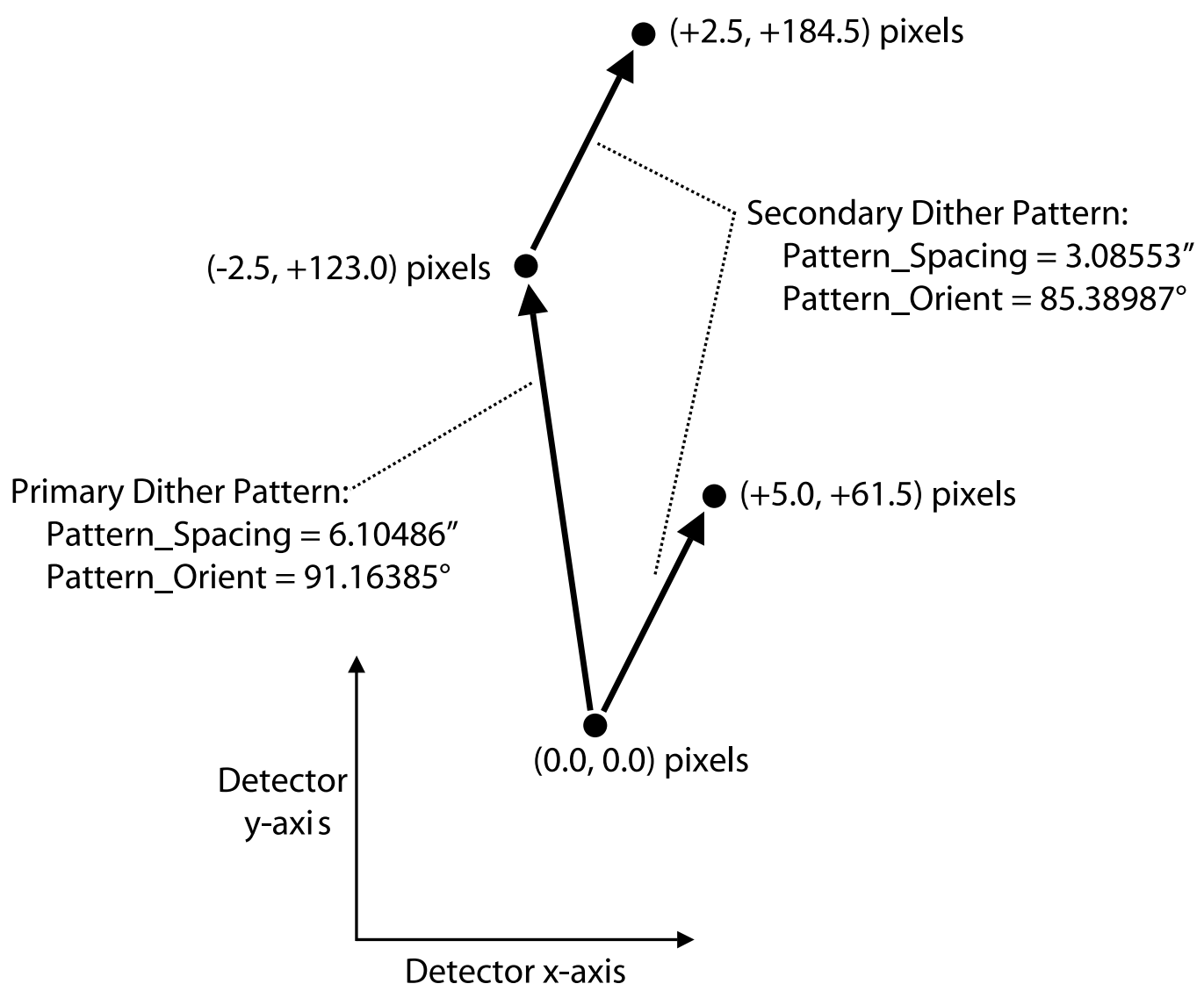

FIG. 2. - Schematic diagram of the COSMOS ACS/WFC four-point dither pattern that we used for each set of four exposures that were obtained for each tile. By combining a two-point primary dither pattern with a two-point secondary pattern at each of the two primary dither points, along a slightly different orientation, each of the four pointings was separated in increments of $\sim 3^{\prime \prime}$ along the $y$-axis of the detector and in increments of $\sim 0.25^{\prime \prime}$ along the $x$-axis of the detector. The Pattern_Spacing and Pattern_Orient parameters shown are those that were used in the phase II file to construct these offsets. This pattern provided a combination of integer-pixel and halfpixel offsets, ensuring that all four pointings would yield good sampling of any point on the sky by all four quadrants of a detector pixel. The distorted geometry of the detector provides an additional modulation of the phase of this half-pixel subsampling across the image, once all four exposures are combined.

with an offset of $\sim 6^{\prime \prime}$, together with a secondary two-point dither pattern at each of the primary dither pattern points, providing an offset of $\sim 3^{\prime \prime}$ along a slightly different orientation. The dither offset spacings and orientations in the two patterns provided the required pattern of half-pixel steps that was needed to ensure good subsampling to the level of $\sim 0.5$ pixels across the entire detector. In Figure 3, we show the effective exposure time obtained after combining all four exposures for an example tile pointing, demonstrating the relatively uniform coverage across the detectors. Specifically, bad columns are moved along the $x$-axis of the detectors, ensuring that these points on the sky are covered by good pixels in three other exposures, and that the gap between the chips is always successfully covered by at least three exposures.

\subsection{ACS Filter Selection}

The primary scientific goal of the COSMOS HST ACS/WFC observations is to obtain the best possible rest-frame optical morphological information on $z \gtrsim 1$ galaxies, thereby necessitating observations at red wavelengths. We chose the broadest filter available on ACS in this wavelength range, namely the F814W ("Broad I") filter. This filter is characterized by having a combination of exceptionally high transmission $(>90 \%-95 \%)$ across an extremely wide wavelength range ( $7300-9500 \AA)$; coupled with the red-sensitive ACS/WFC CCDs, this provided optimal sensitivity to faint rest-frame visible morphological information at the redshifts of interest $(z \gtrsim 1)$.

More specifically, the F814W filter provides an additional $\sim 0.5$ mag of depth relative to the narrower F775W (SDSS $i$ ) filter, for a given exposure time. This is important when comparing the depth of COSMOS to other surveys such as GEMS (Rix et al. 2004), GOODS (Giavalisco et al. 2004), or the UDF (Beckwith et al. 2006), all of which used the SDSS filter set, in particular F775W (SDSS $i$ ) and F850LP (SDSS $z$ ) at the red end of the spectrum, to provide sharp color discrimination through the use of certain spectral break features. This comes at the cost of a shallower depth per unit exposure time than the F814W filter, even though these other fields might be observable for a larger fraction of time with HST, since the total system throughput with the F814W filter is approximately equal to the sum of the F775W and F850LP filters. For this reason, we chose the F814W filter for the COSMOS survey to ensure the deepest possible morphological coverage with $H S T$, while photometry using the SDSS filter set was obtained from ancillary ground-based observations using Subaru (Taniguchi et al. 2007).

For the pilot observations of the central $3 \times 3$ tiles using a bluer filter, we chose the F475W (SDSS $g$ ) filter, motivated by the need to separate extinction-related effects from shape changes 


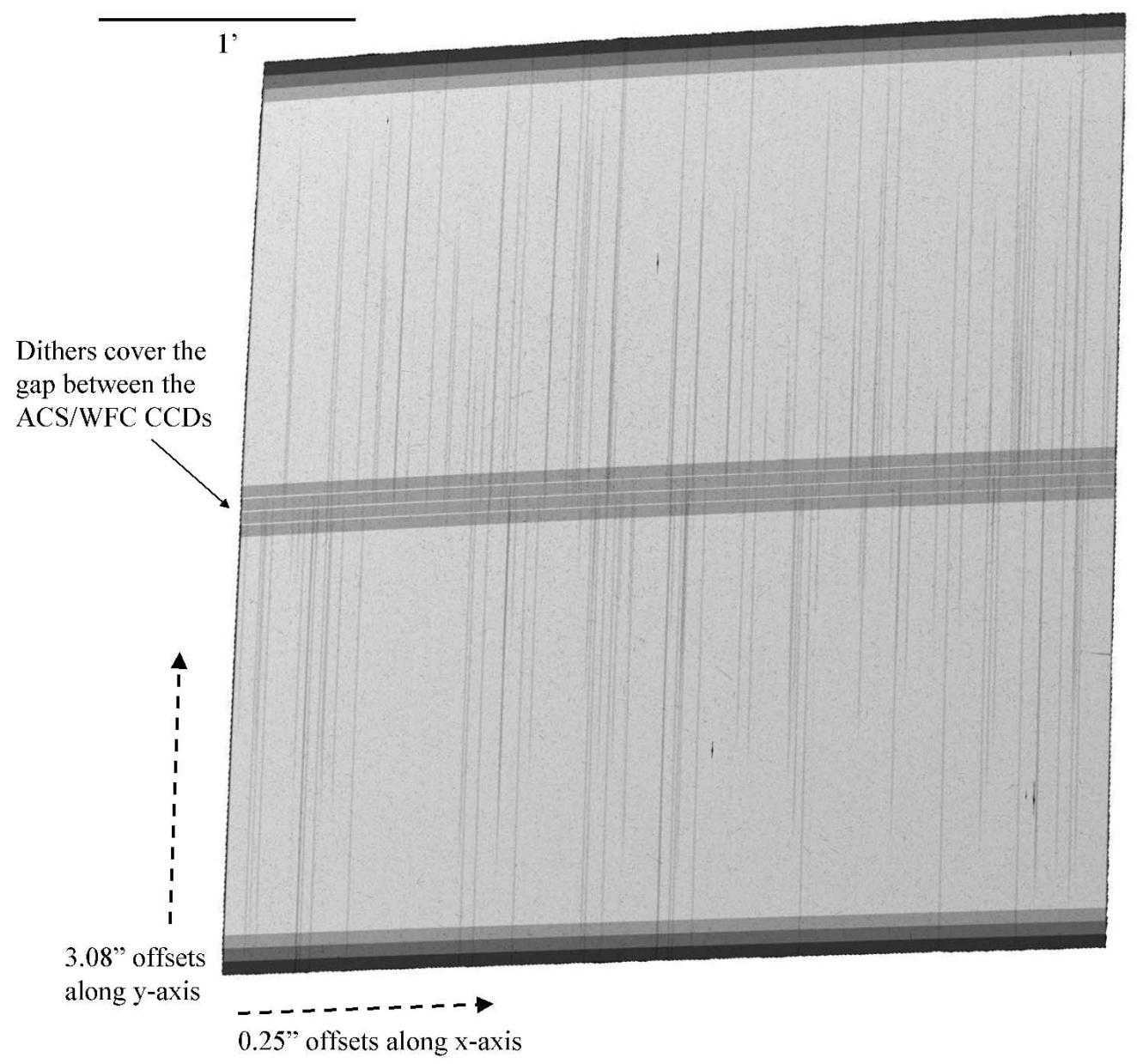

FIG. 3.-Example four-point dither for a single tile, a "weight" image corresponding to the combination of four ACS/WFC exposures of a single pointing, after initial processing through the pipeline. The image is equivalent to effective exposure time, with lighter regions indicating a longer combined exposure time on the final output pixels. Vertical dark lines correspond to bad columns, while the horizontal bands across the center of the image correspond to the $\sim 3^{\prime \prime}$ gap between the two detectors. This image demonstrates that the dither pattern successfully provided coverage of the gap by at least three exposures, as well as moving bad columns along the $x$-direction sufficiently to ensure that they were always covered by good pixels in the other exposures.

introduced by weak lensing. The F475W filter provides optimal throughput in the rest-frame near-UV for our target galaxies at $z \gtrsim 1$, for which weak-lensing studies are the primary goal.

\section{DATA REDUCTION AND PROCESSING}

We processed all the ACS data at STScI on a special-purpose computing cluster purchased especially for the project, consisting of six Linux CPU nodes running pipeline scripts in parallel. As each observation was obtained, the individual exposures were delivered to the computing cluster where they were run through an IRAF/STSDAS pipeline that performed calibration, astrometric registration and cosmic-ray cleaning, as well as final mosaic combination using MultiDrizzle (Koekemoer et al. 2002), which makes use of the DRIZZLE software (Fruchter \& Hook 2002) to remove the geometric distortion and map the input exposures onto a rectified output frame. Here we describe the details of each of these steps.

\subsection{Initial Data Calibration}

Each of the individual $507 \mathrm{~s}$ exposures was first run through the basic steps of ACS calibration using the IRAF/STSDAS task calacs, which performs bias and dark subtraction, gain correction, flat-fielding, and identification of bad pixels. Each data set was retrieved and run through this pipeline as soon as possible after the observation in order to make the data available quickly; this generally necessitated a second-pass calibration a few weeks later with more accurate reference files, in particular using dark and bias reference files that could be created using dark and bias exposures obtained contemporaneously with the data.

After basic calibration, several additional effects also had to be corrected for in the data. In many cases, low-level residual background was present in the images, typically due to scattered light; this was removed by constructing a master scattered-light image for the entire data set through medianing, and then scaling and subtracting this from each individual exposure. In addition, the ACS/WFC CCDs are read out by four amplifiers (two for each CCD), and their bias levels often vary by a few tenths of a count, which is not accounted for in the overscan bias calibration; this was measured and corrected for each exposure.

Finally, the charge transfer efficiency (CTE) of the detectors is gradually deteriorating over time, as a result of charge traps in the pixels created by cosmic-ray hits. This results in lost flux as the charge from each pixel is transferred down the CCD columns during readout; it also produces trails as the traps release their charge after it has passed through. This effect is most severe for pixels that are farthest from the amplifiers, and for faint sources with minimal sky background. Its severity is reduced for sources 
on higher backgrounds ( since there are a finite number of traps in a given pixel, a progressively smaller fraction of the flux is lost for brighter pixels). Ideally, this effect would be corrected for on a pixel-by-pixel basis in the raw images, but this requires a complete physical understanding of the effect, which is still under development. However, the effect can be quantified well enough to apply a postprocessing correction to measured morphological and photometric properties of sources in the images, particularly relevant to the COSMOS weak-lensing studies (Rhodes et al. 2007), based on a knowledge of the source positions and fluxes, together with the sky background values for the exposures involved.

\subsection{Astrometric Image Registration}

After calibration, the next step involved registering the images onto an astrometric grid. The process of aligning the astrometry of HST ACS/WFC images can be separated into two components: (1) ensuring that all four exposures of each tile, obtained during a single orbit, are aligned, and (2) ensuring that adjacent tiles are aligned. Given the small angular scale of the ACS/WFC pixels $\left(0.05^{\prime \prime}\right)$, it is crucial to align images to better than $\sim 2-5$ mas in order to achieve accurate cosmic-ray rejection among the separate exposures within a tile, and to ensure accurate combination of pixels in overlapping regions between adjacent tiles. In addition, it is important to accurately remove the strong distortion that is present in the ACS/WFC images $(\sim 7 \%-10 \%)$; this was done with the MultiDrizzle software (Koekemoer et al. 2002) using distortion solutions provided by Anderson (2005), which are accurate to better than $\sim 0.05-0.1$ pixels across the $4096^{2}$ pixel extent of the $\mathrm{ACS} / \mathrm{WFC}$.

Since each tile was observed during a single orbit, with dither offsets less than $\sim 9^{\prime \prime}$ in total, there was no need for the telescope to change guide stars during the orbit. $H S T$ generally requires two guide stars for accurate tracking; if both guide stars were acquired successfully at the start of the orbit, then those are retained throughout the orbit, and the resulting positional accuracy of small dither offsets is generally known to be better than $\sim 2-3$ mas. This was verified by our processing pipeline, which measured the locations of the sources on each of the four exposures obtained during each orbit and compared the resulting calculated shifts with those which were specified in the Phase II HST observing commands. It was verified that the rms accuracy of relative offsets within an orbit is typically $\lesssim 2$ mas ( $\lesssim 0.04$ pixels); hence, there was generally no significant correction required for these relative offsets within an orbit.

However, a larger astrometric correction is required when aligning adjacent tiles, which is fundamentally due to limitations in the knowledge of the positions of the guide stars. During the cycles when the COSMOS data were obtained (Cycles 12 and 13), all the guide-star positions were still based on the Guide Star Catalog version 1.0, which had no correction for proper motion of guide stars (improved guide-star positions were incorporated into the system after Cycle 14), in addition to many other errors. These effects generally lead to an uncertainty of $\sim 1^{\prime \prime}-2^{\prime \prime}$ or more in the guide-star positions, which is a well-known problem for HST data obtained before Cycle 14. Since the guide-star positions are used to calculate the astrometric information of the exposures, this means that the absolute astrometry of each tile could initially be in error by up to $\sim 1^{\prime \prime}-2^{\prime \prime}$ or more, even though the relative alignment of the four exposures of each tile were accurate to $\lesssim 2$ mas. Moreover, since the two guide stars are typically observed in two different fine guidance sensors (FGSs) separated by $\sim 25^{\prime}$, the uncertainty in their absolute astrometry can translate into an error in the calculated orientation of $H S T$ by as much as $0.01^{\circ}-0.03^{\circ}$ in the worst cases, corresponding to a few pixels across the scale of the $\mathrm{ACS} / \mathrm{WFC}$ detectors, in addition to the error introduced by the uncertainty in the absolute astrometry.

In order to correct the absolute astrometry of the HST ACS/ WFC data sets, we defined a COSMOS astrometric grid (Sanders et al. 2007) based on two ancillary data sets: (1) the first epoch of Very Large Array (VLA) imaging (Schinnerer et al. 2004), to provide a robust fundamental astrometric frame by means of a sufficiently large number of unresolved radio sources across the entire field, and (2) the Canada-France-Hawaii Telescope (CFHT) $i^{*}$-band imaging data set (Capak et al. 2007), which was placed onto the VLA astrometric frame by matching unresolved sources detected in both data sets. The CFHT data in turn provide the best combination of depth and area to enable large numbers of sources to be identified on each ACS/WFC tile for astrometric correction.

On each ACS/WFC tile, there were generally a total of $\sim 300-$ 600 sources identified that were also detected on the CFHT $i^{*}$-band image. The measured right ascension and declination positions of the sources were cross-correlated and matched in order to solve for the transformation between them. Since all the higher order components of the ACS/WFC distortion are removed by the MultiDrizzle software (Koekemoer et al. 2002), the ACS/ WFC tiles can be treated as rectified images, with the only remaining unknown terms being purely linear transformations, specifically an offset in right ascension and declination combined with a possible small rotation, due to the uncertainties in the guide-star positions. Given that the typical astrometric uncertainty in the CFHT images is $\sim 0.1^{\prime \prime}$ for a single source, this can be reduced in quadrature by using as many sources as possible to solve for the transformations, yielding a combined accuracy of $\sim 5$ mas for the resulting shifts when we used the full set of $\sim 300-$ 600 sources on each ACS image that had counterparts in the CFHT images.

This process ensured that all the ACS/WFC exposures and tiles were registered with an absolute astrometric accuracy of $\sim 5$ mas across the entire COSMOS field. These images were placed onto an astrometric frame defined as a tangent plane projection, centered at the nominal COSMOS pointing of R.A. = $10^{\mathrm{h}} 00^{\mathrm{m}} 28.6^{\mathrm{s}}$, decl. $=+02^{\circ} 12^{\prime} 21.0^{\prime \prime} \quad(\mathrm{J} 2000.0)$. The tangent plane projection is the standard projection used for all astronomical images that have a uniform projected pixel size on the sky across the entire image; one of its consequences is that the projected location of a source in the image is slightly farther away from the center than its true angular separation on the sky. For example, at a distance of $30^{\prime}$ from the center, the projected distance of a source in the image is $0.046^{\prime \prime}$ farther away from the center than its true distance. Since all the ancillary COSMOS data sets also use the tangent plane projection, this effect cancels out and is only relevant when comparing the measured position of a source in the COSMOS field to other data that are not on the COSMOS tangent plane, in which case the effect due to the tangent plane projection can be accounted for using simple trigonometry. Throughout this paper, we assume use of the tangent plane projection as described here.

\subsection{Cosmic-Ray Rejection}

Cosmic-ray rejection was carried out primarily among the four exposures within each tile; adjacent information from overlapping tiles was generally not used due to the small amount of overlap. Since each exposure was dithered to a different position on the sky, simple stacking was inadequate to remove cosmic rays, so we used MultiDrizzle to perform the cosmic-ray rejection. This process first used DRIZZLE to remove the distortion from each of the input exposures, mapping them onto four separate output 


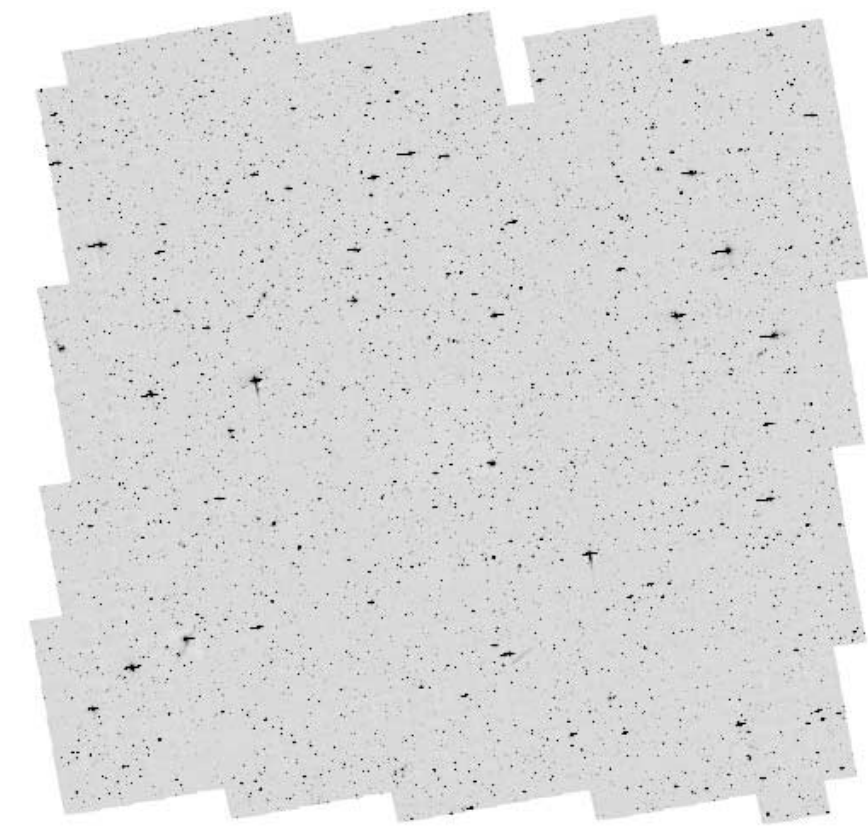

FIG. 4.-Final combined mosaic of all ACS pointings. At a pixel scale of $0.05^{\prime \prime}$ pixel $^{-1}$, this image has 100,800 pixels on a side $\left(\sim 84^{\prime}\right)$, with the observations covering an area of $1.64 \mathrm{deg}^{2}$. The field is centered at R.A. $=10^{\mathrm{h}} 00^{\mathrm{m}} 28.6^{\mathrm{s}}$, decl. $=+02^{\circ} 12^{\prime} 21.0^{\prime \prime}(\mathrm{J} 2000.0)$; the rectangle fully enclosing all the ACS imaging has lower left and upper right corners (R.A., decl. [J2000.0]) at (150.7988 , $\left.1.5676^{\circ}\right)$ and $\left(149.4305^{\circ}, 2.8937^{\circ}\right)$.

images that were aligned to the same pixel grid and rectified, with all the distortion removed, so that all the pixels subtended the same area on the sky.

Once the four drizzled images were created, we used MultiDrizzle to combine them using a median process to create a clean approximation to the final output image. For each $507 \mathrm{~s}$ exposure, $\sim 80,000-250,000$ pixels out of $4096^{2}$ were affected by cosmic rays; therefore, it was rare for a pixel to be affected by cosmic rays during all four exposures ( $\ll 1$ pixel out of $4096^{2}$ ). However, the number of pixels affected by only three cosmic rays out of four exposures was significantly higher (up to $\sim 60$ pixels out of $4096^{2}$ ), and in addition there were many cases where two pixels were affected by cosmic rays, while the third lay on a chip defect or the gap between the chips. In such cases, where three pixels were affected by a defect while the fourth remaining pixel was valid, the median was replaced by the value of the valid pixel if the median value exceeded the valid pixel value by more than $5 \sigma$. This process successfully ensured that we minimized the number of pixels in the resulting median image that might be affected by cosmic rays or chip defects.

Once the clean median image was created for each tile, it was then transformed back to the distorted CCD frame of each of the input exposures, to create a clean version of the input exposure aligned to the original CCD pixel grid. Cosmic rays were then identified using sigma-clipping, comparing the input exposure with the median and calculating the full rms by combing the source counts, background sky counts, and read noise in quadrature. In order to avoid clipping bright stars, the sigma-rejection criterion was softened using a derivative image, which was constructed from the median such that the value of each pixel represented the largest gradient from that pixel to its surrounding neighbors. Pixels were then rejected as cosmic rays only if the difference between the input exposure and the median exceeded the sum of the sigma-criterion and the derivative, scaled by an appropriate factor (thus, the de- rivative component is only significant in bright unresolved sources where it prevents the cores from being clipped, and becomes insignificant in extended or faint sources). The rejection was done in two passes, with the first pass using a $4 \sigma$ clipping combined with a scale factor of 1.2 for the derivative image. The second pass was only performed on pixels surrounding cosmic rays identified during the first pass and was aimed at rejecting fainter pixels associated with the cosmic ray that were not rejected on the first pass, so a more stringent clipping criterion was used ( $3 \sigma$, combined with a scale factor of 0.7 ). The values for this clipping procedure were determined through extensive exploration of parameter space, and are detailed further in Koekemoer et al. (2002).

\section{CONSTRUCTION OF THE FINAL COMBINED TILES AND MOSAIC IMAGES}

Once the cosmic-ray masks had been created, we used MultiDrizzle again, this time combining all the input exposures into a final combined image. For each exposure, we first created a variance map that contained all the components of noise in the image except for the sources themselves, thus containing the sky background (modulated by the flat field and the geometric projection of the detector on the sky), the readout noise, and the dark current, using the measured values applicable to each of the four amplifiers on the ACS/WFC chips. The variance image for each exposure was then inverted and used as a weight map associated with the exposure; inverse variance has the appealing property of scaling linearly with exposure time, and it also has the ability to exclude bad pixels from the drizzle combination by setting their inverse variance weight to zero.

The core of the DRIZZLE algorithm consists of transforming each input-detector pixel onto the output image plane, which may have a different pixel scale and orientation, and distributing the flux from the input pixel among all the output pixels that it may overlap. The PSF in the resulting image is thus convolved three times: by the detector pixel scale (in the original exposure), by the output pixel scale, and again by the detector pixel scale when the pixels are mapped from the input to the output frame. This third convolution can be minimized by shrinking the input pixels by an arbitrary factor (the pixfrac parameter), which ranges between 0 and 1; a pixfrac value of 1 corresponds to simple shiftand-add, thus adding a full convolution by the input pixel size, while a value of 0 corresponds to interlacing, where each input pixel is mapped to a delta function and introduces no additional convolution in the output image plane. After experimentation with different values of pixfrac, we chose a value of 0.8 , which was well matched to our output pixel scale and the degree of subsampling achieved by our dither pattern.

We chose an output pixel scale of $0.03^{\prime \prime}$ pixel $^{-1}$ ( 0.6 times the input CCD detector pixel scale), which not only reduced the convolution by the output pixel size, but also reduced the effects of aliasing that would otherwise result when the input and output pixel scales are comparable. Reducing the aliasing effects had the benefit of providing a more stable PSF for the weak-lensing studies; we also used a Gaussian kernel to produce the images for the lensing studies, which further stabilized the PSF, at the expense of adding some additional correlated noise to the images. For the lensing studies (Rhodes et al. 2007), we produced a combined version of each tile separately, oriented not with north up but rather in the default unrotated frame of the ACS/WFC CCDs, to facilitate processing with the PSF-matching procedures.

For the rest of the COSMOS science projects, we ran MultiDrizzle again to produce additional images, this time oriented with north up, using a square kernel with pixfrac set to 0.8 , and registered with $\sim 5$ mas precision onto the corresponding images 
created from the Subaru and CFHT data (Capak et al. 2007) to facilitate direct comparison between the HST ACS/WFC data and all the other ground-based ancillary data for COSMOS. These images were created at two pixel scales, $0.03^{\prime \prime} \mathrm{pixel}^{-1}$ and $0.05^{\prime \prime} \mathrm{pixel}^{-1}$; we also used the latter set of images to create a single monolithic mosaic file extending for 100,800 pixels along its $x$ and $y$ axes, thus $1.4^{\circ}$ on a side; this image is displayed in Figure 4 . These data products are publicly available through the IPAC/IRSA and STScI/MAST data archive interfaces.

\section{SUMMARY}

We have presented in this paper the details of the HSTACS/WFC COSMOS observations and data processing that we used to produce the imaging data sets which form the basis for the majority of the scientific work in the COSMOS project. A more general discussion of the HST ACS data set is presented in Scoville et al. (2007b). The relative astrometry of all the HST images is accurate to $\sim 5$ mas, with an absolute astrometric accuracy determined fundamentally by the accuracy of the radio reference frame, which is $\sim 55$ mas. The images reach a point-source limiting depth $\mathrm{AB}(\mathrm{F} 814 \mathrm{~W})=27.2$ $(5 \sigma)$ in a $0.24^{\prime \prime}$ diameter aperture, and have been projected onto output grids of $0.03^{\prime \prime}$ pixel $^{-1}$ and $0.05^{\prime \prime}$ pixel $^{-1}$. The $0.03^{\prime \prime}$ pixel $^{-1}$ data were drizzled using a Gaussian kernel with a FWHM of 40 mas, and the average width of the PSF in these images is $0.095^{\prime \prime}$; these images are optimized for weak-lensing studies, and are available both as unrotated tiles (one for each ACS orbit) and as combined sections oriented with north up, registered to the pixel frame of the ground-based optical data sets. The $0.05^{\prime \prime} \mathrm{pixel}^{-1}$ data were drizzled using a square kernel with a pixfrac of 0.8 and have an average PSF width of $0.097^{\prime \prime}$. This data set is available in sections that are registered to the pixel frame of the ground-based optical data sets, and is also available as a single monolithic mosaic 100,800 pixels on a side ( 80 Gbyte). The COSMOS HST data sets are publicly available through the Web sites for IPAC/IRSA ${ }^{11}$ and STScI-MAST. ${ }^{12}$ IRSA also supplies a cutout capability derived from the full-field mosaic, which can be made with any field center and size.

The COSMOS HST Treasury program was supported through NASA grant HST-GO-09822. We wish to thank Tony Roman, Denise Taylor, and David Soderblom for their assistance in the planning and scheduling of the extensive COSMOS observations. We gratefully acknowledge the contributions of the entire COSMOS collaboration, consisting of more than 70 scientists. More information on COSMOS is available at http://cosmos.astro.caltech. edu/. We acknowledge the services provided by the staff at the NASA IPAC/IRSA, as well as the STScI MAST Archive for providing online archive and server capabilities for the COSMOS data sets. The COSMOS Science meeting in 2005 May was supported in part by the NSF through grant OISE-0456439.

11 Available at: http://irsa.ipac.caltech.edu/data/COSMOS/.
Anderson, J. 2005, in HST Calibration Workshop, ed. A. M. Koekemoer, P. Goudfrooij, \& L. L. Dressel (Baltimore: STScI), 11

Beckwith, S. V. W., et al. 2006, AJ, 132, 1729

Capak, P., et al. 2007, ApJS, 172, 99

Davis, M.. et al. 2006, ApJ, 660, L1

Fruchter, A. S., \& Hook, R. N. 2002, PASP, 114, 144

Giavalisco, M., et al. 2004, ApJ, 600, L93

Koekemoer, A. M., Fruchter, A. S., Hook, R. N., \& Hack, W. 2002, in HST Calibration Workshop, ed. S. Arribas, A. M. Koekemoer, \& B. Whitmore (Baltimore: STScI), 337

\section{REFERENCES}

Rhodes, J. R., et al. 2007, ApJS, 172, 203

Rix, H.-W., et al. 2004, ApJS, 152, 163

Sanders, D. B., et al. 2007, ApJS, 172, 86

Schinnerer, E., et al. 2004, AJ, 128, 1974

Scoville, N. Z. et al. 2007a, ApJS, 172, 1 2007b, ApJS, 172, 38

Taniguchi, Y., et al. 2007, ApJS, 172, 9

Williams, R. E., et al. 1996, AJ, 112, 1335 2000, AJ, 120, 2735 